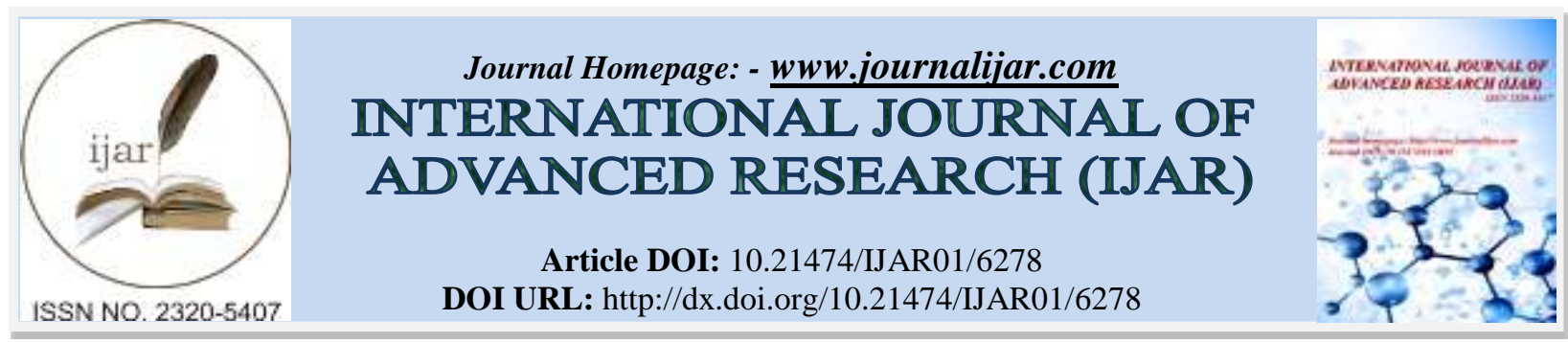

RESEARCH ARTICLE

\title{
THE CUSTOMER PERCEPTION ON WASTE MANAGEMENT OF THE STALLS AT THE MALOLOS FOOD COURT.
}

\author{
Urbano, Kristoffer $\mathbf{P}^{1}$, Simangca, Genelyn ${ }^{2}$, Cruz, Danna May ${ }^{3}$ and Valdez, Janet ${ }^{4}$. \\ 1. La Consolacion University Philippines Graduate School. \\ 2. La Consolacion University Philippines Finance Department. \\ 3. La Consolacion University Philippines Finance Department. \\ 4. La Consolacion University Philippines Graduate School.
}

\section{Manuscript Info}

Manuscript History

Received: 11 November 2017

Final Accepted: 13 December 2017

Published: January 2018

Keywords:-

Customer Perception, Malolos Food

Court, Waste Management.

\begin{abstract}
Solid and liquid wastes generated from the initial extraction of raw materials to the final consumption of products threaten the environment and the people. The absence of proper waste management poses hazards and risks on the health and life of the people, as well as, the preservation of the environment. Steps had been initiated by various governmental and nongovernmental organizations advocating environmental safeguards and proper waste management. From the minuscule step of assessing the small area of food stalls at the Malolos Food Court as to their waste management practices as perceived by their customers, the team of researchers aimed at assessing and creating awareness on public concern and safety on waste management and disposal. The study can be utilized in the formulation of municipal legislation that benefits the customers and the public in general.
\end{abstract}

Copy Right, IJAR, 2018,. All rights reserved.

\section{Introduction:-}

Wastes, solid and liquid, generated from the initial extraction of raw materials up to the final consumption of the finished products accrue to cause adverse effects on the environment and the people. The United States Environmental Protection Agency (2009) warned that the improper handling and disposal of liquid wastes threaten the environment and human beings due to the entrance of these wastes in watersheds, drinking, and potable water.

Parfitt et al. (2010) observed that food wastes discarded from the processes of the preparation of wastes from the serving dishes and guests' plates might be considered edible if not thrown properly. On the other hand, Lim (2010) argued that while the quality of food and service is paramount, a pleasing atmosphere may contribute to even a greater level of overall satisfaction of the customer and his/her subsequent patronage. He stressed that environmental elements in the restaurant have a huge impact on the emotional responses and behavior of customers. These environmental elements include lighting, music, temperature, scent, smell, and furnishing.

The government has taken steps in addressing these concerns on waste management at food stalls. As part of the governmental mandate on public health protection pursuant to the provisions of Republic Act 9711,otherwise known as, the "Food and Drug Administration Act of 2009" and Republic Act 10611, otherwise known as, the "Food Safety Act of 2013", the Food and Drug Administration informs the operators, food handlers of restaurants, catering operations, canteens, and carinderia of the following practical food safety tips to prevent and minimize the risk of 
foodborne illnesses: As to Premises/ Facilities And Sanitation, keep your area clean; protect kitchen areas and food from insects, pests, and other animals; use clean utensils; wash and sanitize all surfaces and equipment used for food preparation; avoid placing pesticides around your kitchen to kill indoor insect and rodent pests; and cleaning chemicals must be kept in suitable labelled containers and stored away from food. Also, as to Basic Personnel Hygiene, wash your hands thoroughly; wash all parts of your hands by rubbing them together vigorously with soap and water for at least 20 seconds in a designated hand washing sink; stay home when sick; and food handlers should stay home when sick with vomiting or diarrhea, or while afflicted with wounds, skin infections, and sores.

Therefore, the public, particularly the customers, are left with the guardianship of role of ensuring that these practices on waste management are followed given the prevention of risks and threats on the safety of the public and environmental preservation.

\section{Statement of the Problem:-}

The study aimed to assess the waste management of Malolos Food Court in the City of Malolos, Bulacan. Specifically, this study sought answers to the following questions:

1. How do the customers perceive the waste management practices on the premises of the food stalls at the Malolos Food Court?

2. How do the customers perceive the waste management practices on the waste disposal of the food stalls at the Malolos Food Court?

3. How do the customers perceive the waste management practices on pest control of the stalls at the Malolos Food Court?

4. How do the customers perceive the waste management practices on sanitation compliance of the stalls at the Malolos City Hall?

5. How does the clientele perceive the waste management practices at the Malolos Food Court?

\section{Methodology:-}

The researchers utilized the quantitative descriptive method of research. This method was designed to gather information about the waste management practices at the Malolos Food Court in the City of Malolos, Bulacan. The descriptive method of research assisted the investigator in knowing how to accomplish the desired purposes at the shortest available time. Moreover, descriptive studies have been used in many areas of investigation for the reason that applies to various kinds of problems (Calmorin, 2001). The respondents of this study included the one hundred (100) regular customers who are mostly students of the Bulacan State University, employees of the Provincial Capitol and the surrounding officers. They were randomly selected to comprise the sampling for the respondents. The study utilized the open-ended questionnaire as the instrument in gathering data. Ogena, Brawner, \& Cristobal (2007), believed that the common method of gathering qualitative data is through an open-ended questionnaire consisting of written questions which the respondents answer. Mcleod (2014) observed that questionnaires are "effective ways of understanding the actions, attitudes, preferences, opinions, and intentions of participants more economically and speedily than other methods

The quantitative data analysis is utilized by the researchers to make sense of or explain the data collected during the research process. Richards (2009) upheld that "suitable data analysis requires one to stay close to his data and interpret it from a position of empathic understanding."

Data analysis in this study commenced with the researcher carefully reading the transcribed data line by line. Data were then divided into meaningful analytical units. The key point coding was used to apply key terms through the detailed examination of passages of text. Codes to be assigned to key points were in the form of words or phrases (Usman, 2011). Coding involves going through the data for themes, ideas, and categories and then marking similar passages of text with a code label so that they can easily be retrieved at a later stage for comparison and analysis (Gibbs \&Taylor, cited in Nicdao, 2015).

\section{Results and Discussions:-}

The customers perceive as 'always' the waste management practices on the premises of the food stalls at the Malolos Food Court, particularly on 'The floor and the kitchen area is regularly swept. 
Table 1:- Waste Management Practices on the Premises of the Stalls in Malolos Food Court as Perceived by the Customers.

\begin{tabular}{|c|c|c|}
\hline Indicators & Mean & Interpretation \\
\hline The floor and kitchen area is regularly swept. & 4.26 & Always \\
\hline No signs of cracks or crumbling walls in the facility. & 4.16 & Often \\
\hline Facilities are regularly painted, fixed or renovated. & 4.04 & Often \\
\hline Average & 4.13 & Often \\
\hline
\end{tabular}

The respondents rated Malolos Food Court regarding premises with a sub-weighted mean of 4.13 which means that they viewed that there are "Often" actions that aim to maintain the cleanliness of the premises/facilities. This is specifically true for the statement "The floor and kitchen area is regularly swept" with a weighted mean of 4.26 or "Always." It is followed by statements "No signs of cracks or crumbling walls in the facility" with a 4.16 weighted mean and "Facilities are regularly painted, fixed or renovated" with a weighted mean of 4.04.

The customers perceive as 'always' the waste management practices on the waste disposal of the food stalls at the Malolos Food Court, particularly on 'The facilities practice reduce, reuse, and recycle method of managing the wastes.'

Table 2:- Waste Management Practices on Waste Disposal of the Stalls in Malolos Food Court as Perceived by the Customers

\begin{tabular}{|c|c|c|}
\hline Indicators & Mean & Interpretation \\
\hline $\begin{array}{l}\text { The facilities practices reduce, reuse and recycle method of } \\
\text { managing the waste. }\end{array}$ & 4.24 & Always \\
\hline $\begin{array}{l}\text { Leftovers are properly disposed so as not affect the quality of } \\
\text { safety of food }\end{array}$ & 4.16 & Often \\
\hline Wastes are regularly collected and are not overflowing. & 4.04 & Often \\
\hline Average & 4.15 & Often \\
\hline
\end{tabular}

The respondents rated the Malolos Food Court with a sub-weighted mean of 4.15 concerning waste disposal which means that they viewed that there are "Often" actions that aim to maintain proper waste disposal at the stalls in the food court. It is specifically stated in "The facilities practices reduce, reuse and recycle method of managing the waste" with a weighted mean of 4.24 or "Always" followed by statements "Left-overs are properly disposed so as not to affect the quality of safety of food" with 4.16 weighted mean and "Wastes are regularly collected and are not overflowing" with 4.04 weighted mean.

The customers perceive as 'often' the waste management practices on the pest control of the food stalls at the Malolos Food Court, particularly on 'No signs of cockroaches and rats are running around in the facility; No known instances of students or staff that have complained of being afflicted with dengue or malaria at the Malolos Food Court; and No signs of insects or other pests are seen in the facility.

Table 3:- Waste Management Practices on Pest Control of the Stalls in Malolos Food Court as Perceived by the Customers

\begin{tabular}{|c|c|c|}
\hline Indicators & Mean & Interpretation \\
\hline $\begin{array}{l}\text { No signs of cockroaches and rats are running around in the } \\
\text { facility. }\end{array}$ & 3.88 & Often \\
\hline $\begin{array}{l}\text { No known instances of students or staffs that have complained } \\
\text { of being afflicted with dengue or malaria in the Malolos Food } \\
\text { Court. }\end{array}$ & 3.96 & Often \\
\hline No signs of insects or other pests were seen in the facility. & 4.10 & Often \\
\hline Average & 3.98 & Often \\
\hline
\end{tabular}

The respondents rated the Malolos Food Court with a sub-weighted mean of 3.98 regarding pest control which means that they viewed that there are "Often" actions that aim to maintain pest control of the food court. It is stated in the "No signs of insects or other pests were seen in the facility" with a weighted mean of 4.10 while the "No known instances of students or staffs that have complained of being afflicted with dengue or malaria in the Malolos 
Food Court" with 3.96 weighted mean and "No signs of cockroaches and rats are running around in the facility" with weighted mean of 3.88 .

The customers perceive as 'always' the waste management practices on the sanitation compliance of the food stalls at the Malolos Food Court, particularly on 'The eatery have their sanitation and registration compliance displayed; Staff have trimmed fingernails and wear minimum requirement for proper attire in the kitchen, such as apron and hairnet; and There are unknown instances of someone being food poisoned at the Malolos Food Court.

Table 4:- Waste Management Practices on Sanitation Compliance of the Stalls in Malolos Food Court as Perceived by the Customers

\begin{tabular}{|l|c|c|}
\hline Indicators & Mean & Interpretation \\
\hline $\begin{array}{l}\text { The eatery have their sanitation and registration compliance } \\
\text { displayed. }\end{array}$ & 4.34 & Always \\
\hline $\begin{array}{l}\text { Staffs have trimmed fingernails and wear minimum requirement } \\
\text { for proper attire in the kitchen such as apron and hairnet. }\end{array}$ & 4.32 & Always \\
\hline $\begin{array}{l}\text { There are unknown instances of someone being food poisoned } \\
\text { in the Malolos Food Court. }\end{array}$ & 4.30 & Always \\
\hline Average & $\mathbf{4 . 3 2}$ & \multicolumn{1}{l}{} \\
\hline
\end{tabular}

The respondents rated the Malolos Food Court with a sub-weighted mean of 4.32 regarding sanitation compliance which means that they viewed that there are "Always" actions that aim to comply with the sanitation standard of the food court. It is specifically stated "The eatery have their sanitation and registration compliance displayed." with a weighted mean of 4.34. The sanitation compliance on the "Staffs have trimmed fingernails and wear minimum requirement for proper attire in the kitchen such as apron and hairnet" have a 4.32 weighted mean and "There are unknown instances of someone being food poisoned in the Malolos Food Court" with a weighted mean of 4.30.

The customers of the Malolos Food Court who are students and staff from the Bulacan State University, as well as, the staff from the Provincial Capitol and the surrounding offices have positive perception on the waste management practices at the Malolos Food Court as manifested in their answers to the questionnaire. They believe that the food stalls adhere to proper and healthful waste management practices. The positive perception can, also, be confirmed from the continuous multitude patronage of the public despite the open-space location, the absence of air conditioning units, and other amenities comparable to the food stalls at the malls.

\section{Conclusions and Recommendations:-}

Based on the results and findings of the study, the researchers present the following conclusions and recommendations.

1. The waste management practices of the food stalls at the Malolos Food Court comply with the sanitary requirements which adhere to the environmental preservation and public safety.

2. The Malolos Food Court has high compliance with their sanitation and waste management practices considering that the landowner of the facility is the Provincial Government of Bulacan.

3. There is a need to look into the indicators in which the food stalls at the Malolos Food Court were not perceived to have 'always' maintained waste management practices.

4. The indicators which were not 'always' practiced could be utilized in the improvement of the waste management of the food stalls at the Malolos Food Court.

5. The results and findings of this study can be utilized in the formulation of necessary legislation and municipal laws to enhance the governmental services to public health and safety.

\section{References:-}

1. Muralisrinivasan Subramanian, N. (2016). Plastics Waste Management: Processing and Disposal. Shawbury, Shrewsbury, Shropshire, U.K.: Smithers Rapra.

2. http://ezinearticles.com/?Garbage-Disposal-System-For-Proper-WasteManagement\&id=4432903

3. https://theses.lib.vt.edu/theses/available/etd-07232012-

4. https://www.researchgate.net/publication/265387304_Solid_waste_management_in_the_hospitality_industry_A _review. 